\title{
Quality of life in type 2 diabetes mellitus patients requiring insulin treatment in Buenos Aires, Argentina: a cross-sectional study
}

\author{
Andres Pichon-Riviere ${ }^{1,2}$, Vilma Irazola ${ }^{1}$, Andrea Beratarrechea ${ }^{1,3}$, Andrea Alcaraz $^{1}$, Carolina Carrara ${ }^{1,4^{*}}$
}

\begin{abstract}
Background: Decision-makers have begun to recognize Health-Related Quality of Life (HRQoL) as an important and measurable outcome of healthcare interventions; and HRQoL data is increasingly being used by policy-makers to prioritize health resources. Our objective was to measure HRQoL in a group of Type 2 Diabetes Mellitus (T2DM) patients receiving insulin treatment in Buenos Aires, Argentina.

Methods: We conducted a cross-sectional study of patients with T2DM over 21 years of age, treated with either Neutral Protamine Hagedorn (NPH) insulin or Insulin Glargine (IG), who had not changed their baseline schedule in the last 6 months. The recruitment was during 2006-7 in nine private diabetes specialists' offices in Buenos Aires, Argentina. A standardized diabetes-specific HRQoL questionnaire, the Audit of Diabetes Dependent Quality of Life (ADDQoL), was used.

Results: A total of 183 patients were included (93 receiving NPH and 90 receiving IG). The mean QoL score was: 0.98 (SD: 0.89) and the diabetes specific QoL was: -1.49 (SD: 0.90). T2DM had a negative impact on HRQoL with a mean Average Weighted Impact (AWI) score on QoL of -1.77 (SD: 1.58). The greatest negative impact was observed for domains: 'worries about the future', 'freedom to eat', 'living conditions', 'sex life', and 'family life'. The mean AWI score was -1.71 (SD: 1.48) in patients treated with IG and -1.85 (SD: 1.68) in patients receiving NPH, this difference was not statistically significant.

Conclusion: The ADDQoL questionnaire is a tool that can be used in Argentina to measure the QoL of patients with diabetes when evaluating diabetes care programs. The scores of QoL in our selected population did not differ from those reported in high-income countries. We expect that the results of this study will increase healthcare providers' awareness of patients' perceived QoL and help to overcome the barriers that delay insulin treatment; mainly clinical inertia and patient resistance.

Keywords: Quality of Life (QoL), Type 2 Diabetes Mellitus (T2DM), Audit of Diabetes Dependent Quality of Life (ADDQoL), Argentina

Copyright: (C) 2015 by Kerman University of Medical Sciences

Citation: Pichon-Riviere A, Irazola V, Beratarrechea A, Alcaraz A, Carrara C. Quality of life in type 2 diabetes mellitus patients requiring insulin treatment in Buenos Aires, Argentina: a cross-sectional study. Int J Health Policy Manag. 2015;4(7):475-480. doi:10.15171/ijhpm.2015.80
\end{abstract}

\section{Article History:}

Received: 11 November 2014

Accepted: 6 April 2015

ePublished: 10 April 2015

\section{Key Messages}

Implications for policy makers

- The Audit of Diabetes Dependent Quality of Life (ADDQoL) questionnaire is a tool that can be used in Argentina to measure the QoL of patients with diabetes when evaluating diabetes care programs.

- The assessment of patient-reported outcomes could be easily incorporated into ambulatory practice and can provide valuable information when evaluating diabetes care programs.

- While diabetes adversely affects QoL, patients treated with insulin in middle-income countries can maintain a high QoL similar to that observed in patients with diabetes in high-income countries.

- This can be a valuable argument to help overcome barriers that delay insulin treatment, like clinical inertia and patient resistance.

Implications for public

Nowadays, it is unanimously recognized that Health-Related Quality of Life (HRQoL) is an important and measurable outcome of healthcare interventions. Increasingly, health professionals and policy-makers are using HRQoL data to make decisions about treatment options and prioritization of health resources. As expected, our study confirmed that diabetes negatively affects the QoL of patients in Argentina. However, while the impact on QoL was similar to that reported by studies in other parts of the world, our study patients, all treated with insulin, were able to maintain QoL levels comparable to those of patients in high-income countries. As insulin is one of the most effective measures to achieve diabetes control, this should be a good reason not to delay insulin treatment when indicated. 


\section{Background}

Traditionally, the impact of a chronic disease has been measured in terms of either morbidity or mortality. However, researchers have begun to recognize Health-Related Quality of Life (HRQoL) as an important and measurable outcome of healthcare interventions. In patients with diabetes, it may predict an individual's capacity to manage this condition and to maintain long-term health and well-being (1). Importantly, HRQoL data is also used by policy-makers to make decisions, particularly in regards to resource allocation for diabetes and other chronic diseases $(2,3)$.

Diabetes Mellitus (DM) negatively impacts HRQoL. This negative impact affects multiple aspects of a person's life, including the psychological impact of being chronically ill, dietary restrictions, changes in social life, symptoms of inadequate metabolic control, chronic complications and ultimately lifelong disabilities (3-8). Many variables have been associated with HRQoL in patients with DM: age, gender, socio-economic status, obesity, type of DM, treatment, chronic complications, health insurance, quality of care and patient education (4,9-12).

In Argentina, high rates of physical inactivity, unhealthy diets and obesity have led to an increase in the prevalence of DM. The rates of DM increased from $8.4 \%$ in 2005 to $9.6 \%$ in 2009 (13).

Several studies have shown unnecessary delays in the initiation of insulin therapy, especially in developing countries. Clinical inertia as well as patients' concerns about the potential negative impact of insulin therapy on their QoL are the main barriers to the timely initiation of this treatment (14).

Despite its importance, there is no information about QoL in patients with Type 2 Diabetes Mellitus (T2DM) receiving insulin therapy in Argentina.

The primary objective of this study was to measure HRQoL in a group of T2DM receiving insulin treatment in Buenos Aires, Argentina, through a standardized diabetes-specific HRQoL questionnaire, the Audit of Diabetes Dependent Quality of Life (ADDQoL). The secondary objective was to explore variables related to HRQoL in these patients.

\section{Methods}

We performed a cross-sectional study to describe HRQoL in a group of patients suffering from T2DM. A consecutive sample of eligible patients with T2DM, treated by diabetes specialists at private medical offices in Buenos Aires, Argentina, was taken for this study. We included patients with T2DM over 21 years of age, treated with either Neutral Protamine Hagedorn $(\mathrm{NPH})$ insulin or Insulin Glargine (IG), who had not changed their baseline schedule in the last 6 months. They were invited to participate during their usual doctor's visit. We included 9 private doctor's offices. At the time of the recruitment, during 2006-7, IG and NPH were the only insulin baseline treatment schemes available in Argentina. Although the proportion of patients receiving NPH in Argentina was greater than that of those receiving IG, the use of long-acting basal insulin analogues was growing rapidly in the country.

Pregnant and lactating women and patients with any unstable medical illness, mental disease, the inability to read or write and those who chose not to participate in the study were excluded.

\section{Assessment of Quality of Life (QoL)}

We used the validated Spanish version for Argentina of the ADDQoL. The original version of the ADDQoL in English was translated and transculturally adapted to Argentina by Perrotta and Irazola (15) showing an adequate reliability and validity. The ADDQoL has been designed to allow users to rate the impact of diabetes on different domains, together with the perceived importance of each domain on their HRQoL (16). This instrument consists of 20 items dealing with 18 life domains. The individual items are formulated in the format of "If I did not have diabetes my [life domain] would be": (very much better/much better/a little better/the same/worse/much worse/very much worse). Each item is scored on a 7-point scale ( -3 maximum negative impact of diabetes to +3 maximum positive impact of diabetes). Then, the respondent indicates whether the item is very important, important, quite important, or not at all important for him or her ( 3 to 0 ). The respondent is allowed to indicate if an item is not applicable to his or her condition. Scoring and importance are multiplied for the applicable items and averaged to determine the final score (range - 9 maximum negative impact of diabetes to +9 maximum positive impact of diabetes). An Average Weighted Impact (AWI) score is derived by adding the weighted impact scores for each domain and dividing by the number of applicable domains. The AWI score can range from -9 (maximum negative impact) to +9 (minimum negative impact).

Life domains included in the questionnaire were: Work life and work-related opportunities, family life, friendship and social life, sex life, physical appearance, physical health, holidays or leisure activities, local or long distance journeys, confidence to do things, motivation to achieve things, the way society reacts, worries about the future, financial situation, unwanted dependence on others, living conditions, freedom to eat, enjoyment of food, and freedom to drink [respondents may indicate Not Applicable (NA) as a response for work life and work-related opportunities, family life and sex life domains]. In addition to the assessment of these domains, the ADDQoL also includes two global measures. The first one evaluates the global Quality of Life (present QoL) "In general, my present QoL is": (excellent/very good/good/neither good nor $\mathrm{bad} / \mathrm{bad} /$ very bad/extremely bad) (range -3 to +3 ). The second evaluates the global impact of diabetes on QoL (QoL specific diabetes) in the format "If I did not have diabetes my QoL would be": (very much better... very much worse) (range -3 to +3$)$.

We collected socio-demographic data (gender, level of education, health coverage), diabetes complications and associated conditions (duration, presence of macrovascular and/or microvascular complications, other chronic conditions such as hypertension, hyperlipidemia or obesity), diabetes therapy and other medications for chronic conditions, diabetes education [according to QUALIDIAB criteria (17)], compliance with diet and physical exercise, clinical parameters (body weight, height) and laboratory measurements (glycaemia, glycosylated hemoglobin, cholesterol total, Lowdensity and high-density lipoprotein, triglycerides, creatinine and microalbuminuria). 


\section{Sample size}

We estimated a sample size in order to obtain 95\% Confidence Intervals (CI) of the QoL score with an error estimation of 0.30 points (semi-amplitude). Based on previous studies $(16,18,19)$, we anticipated a QoL mean score in T2DM patients of $1.20 \pm 1.30$ (mean \pm SD). We included an additional $20 \%$ to the total sample to account for the possibility of loss of follow up or incomplete data based on experiences of previous field work conducted by our research group. The total sample size for our study based on these criteria required 90 T2DM patients treated with NPH insulin and 90 with IG.

\section{Statistical analysis}

Descriptive statistics were calculated for all variables measured. We used the Chi-Square test to explore the association between categorical variables. For continuous variables, we used the independent-sample T-Test or the Wilcoxon rank-sum test according to the distribution of the analysed variables. $P$-values of less than 0.05 (two-tailed) were considered to be statistically significant. We calculated the mean, standard deviation and median of the ADDQoL measures and performed subgroup analyses to examine the association of socio-demographic characteristics, level of glycemic control and diabetes complications with ADDQoL scores. Additionally, three robust regression models were built in order to adjust for potential confounders. Model selection was performed following a stepwise approach. Linearity was tested through augmented component-plusresidual plots. Outliers and potential influence were explored by calculating studentized residuals and Cook's distance for each observation. The reliability of the scale was determined by assessing the Cronbach alpha coefficient. Item missing rate was used to check feasibility. Data analyses were performed using Stata version 8.0 (Stata Corp, College Station, TX, USA).

\section{Results}

\section{Patient characteristics}

A total of 206 patients were invited to participate. Twentythree $(11 \%)$ did not give their consent to participate or were excluded because of incomplete data. A total of 183 patients met the inclusion criteria and provided complete data for the analysis (93 receiving NPH and 90 receiving IG). Data on respondent characteristics are presented in Table 1.

\section{Quality of Life (QoL)}

The scale showed a satisfactory feasibility since the item missing values were lower than $2 \%$. Analyses of reliability showed a high internal consistency (Cronbach alpha: 0.89). The mean present QoL score was 0.98 (SD: 0.89), the diabetes specific QoL was -1.49 (SD: 0.90) and the mean AWI score on QoL -1.77 (SD: 1.58), indicating an overall negative impact of DM on QoL in T2DM patients treated with insulin.

When we explored the association of socio-demographic characteristics, level of glycemic control and diabetes complications with ADDQoL scores (Table 2), we only found significant differences in the analysis by gender. In the crude analysis, Present QoL was significantly better in men than women [mean: 1.25 (SD: 0.77) vs. 0.72 (SD: 0.95) $P<0.01$ ]. This difference remained significant in the adjusted analysis (partial beta coefficient $=-0.48 ; 95 \%$ CI: -0.84 to -0.12 ;
Table 1. Patient characteristics $(n=183)$

\begin{tabular}{|c|c|}
\hline Variables & \\
\hline Female, $\mathrm{n}(\%)$ & $88(48.09)$ \\
\hline Age (years), median (interquartile range) & $65(55-73)$ \\
\hline Employed, n (\%) & $86(46.99)$ \\
\hline Health insurance, $\mathrm{n}(\%)$ & $180(98.36)$ \\
\hline$\geq 12$ years of formal education, $n(\%)$ & $104(56.83)$ \\
\hline Adequate Diabetes education ${ }^{\mathrm{a}}, \mathrm{n}(\%)$ & $176(96.17)$ \\
\hline Duration of diabetes in years, mean $\pm S D$ & $12.74 \pm 8.30$ \\
\hline $\mathrm{HbA1c}(\%)$, mean $\pm \mathrm{SD}$ & $7.59 \pm 1.97$ \\
\hline $\mathrm{HbA} 1 \mathrm{c} \geq 8 \%^{\mathrm{b}}, \mathrm{n}(\%)$ & $59(32.24)$ \\
\hline Bolus treatment with regular insulin, $\mathrm{n}(\%)$ & $139(75.96)$ \\
\hline \multicolumn{2}{|l|}{ Basal treatment } \\
\hline NPH insulin, $\mathrm{n}(\%)$ & $93(50.82)$ \\
\hline IG, n (\%) & $90(49.18)$ \\
\hline Number of daily insulin injections, mean \pm SD & $3.64 \pm 1.61$ \\
\hline Oral hypoglycemic agents, $\mathrm{n}(\%)$ & $82(44.81)$ \\
\hline Macroangiopathic complications, n (\%) & $51(27.87)$ \\
\hline Microangiopathic complications, n (\%) & $49(26.78)$ \\
\hline \multicolumn{2}{|l|}{ Acute complications } \\
\hline Diabetes-related hospitalizations, n (\%) & $6(3.28)$ \\
\hline Severe hypoglycemia, n (\%) & $7(3.83)$ \\
\hline Ketoacidosis, n (\%) & $1(0.54)$ \\
\hline Hyperosmolar syndrome, n (\%) & $0(0.00)$ \\
\hline Hypertension, n (\%) & $124(67.76)$ \\
\hline Hypercholesterolemia, n (\%) & $97(53.00)$ \\
\hline Current smokers, n (\%) & $25(13.66)$ \\
\hline $\mathrm{BMI} \geq 30, \mathrm{n}(\%)$ & $71(38.80)$ \\
\hline
\end{tabular}

$\mathrm{BMI}=$ Body Mass Index; $\mathrm{HbA} 1 \mathrm{C}=$ Glycosylated Hemoglobin; $\mathrm{IG}=$ Insulin Glargine.

${ }^{a}$ According to QUALIDIAB criteria (17).

${ }^{b} \mathrm{HbA} 1 \mathrm{C} \geq 8 \%$ was defined as poor control.

$P<0.01)$. There was no difference in the DM specific QoL and AWI score.

The mean AWI score was -1.71 (SD: 1.48) in the group of patients treated with IG and -1.85 (SD: 1.68 ) in the group of patients receiving NPH. This difference was not statistically significant. Likewise, for the remaining variables analyzed, we did not find statistically significant differences in the ADDQoL scores either in the bivariate analysis or after adjusting for age, gender, education, employment status, health insurance, obesity, Glycosylated Hemoglobin (HA1c) and diabetes complications.

In our analysis of the impact of diabetes on QoL for different domains (Table 3 and Figure 1), the greatest negative impact of T2DM was observed for the domain 'worries about the future' with a mean AWI score -2.54 (SD: 3.63). The other domains where ADDQoL showed a greater effect on HRQoL were, 'freedom to eat as I wish' -2.47 (SD: 2.49)', 'living conditions' -2.33 (SD: 2.63), 'sex life' -2.27 (SD: 2.60), and 'family life' -2.05 (SD: 2.46).

The domains 'sex life' and 'enjoyment of food' were more affected in men than in women with a mean -2.96 (SD: 2.51) vs. -1.38 (SD: 2.49) $P<0.01$; and -1.98 (SD: 2.33 ) vs. -1.21 (SD: 2.45) $P=0.03$, respectively. Alternatively, the domains 'living conditions' and 'friendship and social life' were more affected in women than in men with a mean -2.82 (SD: 2.55) vs. -1.89 (SD: 2.67) $P<0.01$; and -1.49 (SD: 2.14) vs. -0.98 (SD: 2.08) $P=$ 
Table 2. ADDQoL scores according to patient characteristics

\begin{tabular}{|c|c|c|c|}
\hline \multirow{2}{*}{$\begin{array}{l}\text { Variables } \\
\text { Gender }\end{array}$} & \multicolumn{2}{|c|}{ ADDQoL scores (Mean \pm SD) } & \multirow[t]{2}{*}{$P$-value ${ }^{a}$} \\
\hline & Male & Female & \\
\hline Present QoL & $1.25 \pm 0.77$ & $0.72 \pm 0.95$ & $<0.01$ \\
\hline DM specific QoL & $-1.49 \pm 1.25$ & $-1.23 \pm 1.11$ & 0.50 \\
\hline AWI & $-1.76 \pm 1.69$ & $-1.79 \pm 1.46$ & 0.28 \\
\hline Age & $\geq 65$ years & $<65$ years & \\
\hline Present QoL & $0.98 \pm 0.93$ & $0.98 \pm 0.86$ & 0.73 \\
\hline DM specific QoL & $-1.50 \pm 0.91$ & $-1.49 \pm 0.90$ & 0.64 \\
\hline AWI & $-1.85 \pm 1.79$ & $-1.72 \pm 1.34$ & 0.23 \\
\hline $\mathrm{BMI} \geq 30$ & Yes & No & \\
\hline Present QoL & $1.41 \pm 1.43$ & $1.34 \pm 0.98$ & 0.70 \\
\hline DM specific QoL & $-1.49 \pm 0.88$ & $-1.50 \pm 0.93$ & 0.76 \\
\hline AWI & $-1.66 \pm 1.41$ & $-1.84 \pm 1.69$ & 0.52 \\
\hline Basal treatment & Glargine & $\mathrm{NPH}$ & \\
\hline Present QoL & $1.03 \pm 0.94$ & $0.94 \pm 0.86$ & 0.87 \\
\hline DM specific QoL & $-1.45 \pm 0.98$ & $-1.54 \pm 0.83$ & 0.97 \\
\hline AWI & $-1.71 \pm 1.48$ & $-1.85 \pm 1.68$ & 0.87 \\
\hline HbA1C (\%) & $\geq 8$ & $<8$ & \\
\hline Present QoL & $1.25 \pm 1.21$ & $1.54 \pm 1.12$ & 0.13 \\
\hline DM specific QoL & $-1.49 \pm 0.87$ & $-1.48 \pm 0.92$ & 0.52 \\
\hline AWI & $-1.71 \pm 1.52$ & $-1.80 \pm 1.62$ & 0.33 \\
\hline Diabetes complications & Yes & No & \\
\hline Present QoL & $1.36 \pm 1.12$ & $1.35 \pm 1.21$ & 0.97 \\
\hline DM specific QoL & $-1.52 \pm 0.92$ & $-1.47 \pm 0.89$ & 0.53 \\
\hline AWI & $-1.87 \pm 1.55$ & $-1.70 \pm 1.61$ & 0.34 \\
\hline $\begin{array}{l}\geq 12 \text { years of formal } \\
\text { education }\end{array}$ & Yes & No & \\
\hline Present QoL & $1.08 \pm 0.91$ & $0.82 \pm 0.86$ & 0.83 \\
\hline DM specific QoL & $-1.47 \pm 0.86$ & $-1.53 \pm 0.85$ & 0.69 \\
\hline AWI & $-1.60 \pm 1.43$ & $-2.02 \pm 1.74$ & 0.80 \\
\hline Employed & Yes & No & \\
\hline Present QoL & $1.07 \pm 0.77$ & $0.87 \pm 1.03$ & 0.30 \\
\hline DM specific QoL & $-1.53 \pm 0.97$ & $-1.49 \pm 0.95$ & 0.49 \\
\hline AWI & $-1.54 \pm 1.14$ & $-2.00 \pm 1.92$ & 0.76 \\
\hline
\end{tabular}

ADDQoL= Audit of Diabetes Dependent Quality of Life; QoL= Quality of Life; DM= Diabetes Mellitus; AWI: Average Weighted Impact; NPH= Neutral Protamine Hagedorn; BMI= Body Mass Index; HbA1C= Glycosylated Haemoglobin.

a $P$-values correspond to the multivariate analysis.
Table 3. Weighted impact scores for QoL domains in T2DM patients

\begin{tabular}{lccc}
\hline Life domain & Mean & SD & Median \\
\hline Worries about the future & -2.54 & 3.63 & -2 \\
Freedom to eat as I wish & -2.47 & 2.49 & -2 \\
Living conditions & -2.33 & 2.63 & -2 \\
Sex life & -2.27 & 2.60 & -2 \\
Family life & -2.05 & 2.46 & -2 \\
The things I could do physically & -1.98 & 2.44 & -2 \\
Ease of traveling (local or long distance) & -1.88 & 2.40 & -1 \\
Holidays or leisure activities & -1.85 & 2.40 & -1 \\
Work life and work-related opportunities & -1.83 & 2.55 & 0 \\
Motivation to achieve things & -1.76 & 2.46 & -1 \\
Confidence in my ability to do things & -1.71 & 2.17 & -2 \\
Enjoyment of food & -1.64 & 2.46 & -1 \\
Physical appearance & -1.48 & 2.37 & -1 \\
Finances & -1.42 & 2.16 & 0 \\
Unwanted dependence on others & -1.24 & 2.85 & 0 \\
Friendship and social life & -1.23 & 2.12 & 0 \\
Freedom to drink as I wish & -1.11 & 2.15 & 0 \\
The way society reacts to me & -0.87 & 2.03 & 0 \\
\hline QoL Qualty of Life; T2D & &
\end{tabular}

QoL= Quality of Life; T2DM= Type 2 Diabetes Mellitus.

0.03 , respectively. People younger than 65 years old showed a lower score than the elderly in the domain 'worries about the future' [mean: -3.16 (SD: 3.69 ) vs. -1.98 (SD: 3.55); $P=0.01$ ].

Discussion

This is the first study describing HRQoL in T2DM patients treated with insulin using a diabetes specific instrument as ADDQoL in Argentina. Our results showed that T2DM has a negative impact on HRQoL in a group of Argentinean diabetes patients treated with insulin. However, mean AWI scored were not significantly different between patient treated with NPH or IG. As stated by Polonsky, the relationship between HRQoL and diabetes is bidirectional; aspects of diabetes may negatively impact on HRQoL, and impaired HRQoL may also negatively influence diabetes self-management and thus health outcomes (20). Therefore, it is relevant to explore and understand those factors implicated in this relationship to

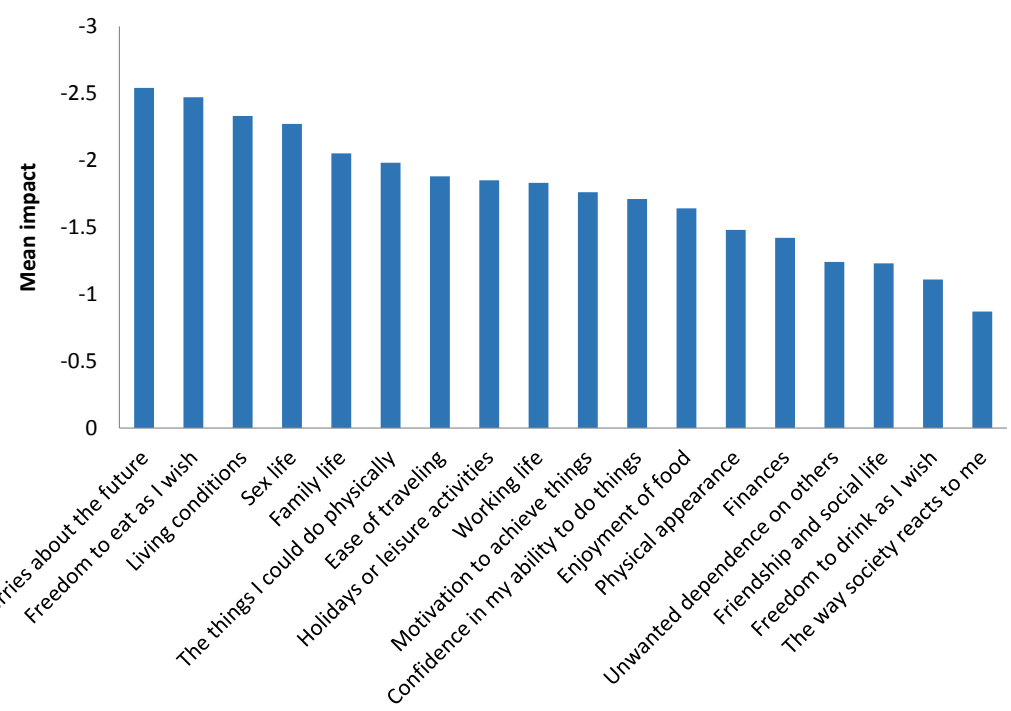

Figure 1. Impact of diabetes on individual life domains. 
improve T2DM outcomes.

Some studies found that better glycemic control was associated with better HRQoL and that complications were the most important disease-specific determinant of HRQoL $(4,21,22)$. However, other authors found no significant relationship between HRQoL and glycemic control (2325). In our study, glycemic control was not associated with HRQoL while patients with complications showed lower QoL scores than patients without complications, though this difference was not statistically significant. Additionally, men with T2DM reported higher scores in present QoL than women, a finding consistent with other studies in similar populations $(9,12,26-29)$.

Several studies showed that the most negatively impacted domains were those related to food, namely, 'freedom to eat', 'enjoyment of food' and 'freedom to drink'. Additionally, as found in our sample, 'worries about the future' is also one of the most affected domains (10,16,30-32). As expected, the negative impact of diabetes varies depending on the characteristics of the patients. For example, the domain 'worries about the future' had particularly large negative impact on HRQoL, in the subgroup of subjects younger than 65 years old. Collins et al. (30) showed that older age (60 years of age) might be associated with higher diabetes-related QoL scores, although this statistically significant association was diminished after adjusting for relevant factors. Sundaram et al. (33) also reported that older age (60 years of age) was independently associated with higher ADDQoL scores in a multivariate analysis. Differences between genders were also seen in domains such as sex life, friendship and social life and enjoyment of food, which is consistent with findings in other studies $(9,26)$.

Our main limitation is that the study sample was drawn from specialized diabetes clinics rather than randomly selected centers, affecting the generalizability of our findings. Patients treated in specialist clinics usually have high health coverage and higher levels of diabetes education than those T2DM patients treated in public hospitals with no health coverage. On the other hand, the relatively small sample size did not allow us to further explore the potential association of QoL with socio-demographic characteristics and different aspects of the disease.

\section{Conclusion}

The ADDQoL questionnaire is a tool that can be used in Argentina to measure the QoL of patients with diabetes when evaluating diabetes care programs. The scores of QoL in our selected T2DM patient population did not differ from those reported in studies that included patients from high-income countries where there is access to a high level of diabetes care. While diabetes adversely affects QoL, patients treated with insulin in middle-income countries can maintain a high QoL similar to that observed in patients with diabetes in highincome countries. In future studies, it would be advisable to implement this questionnaire in a different patient population, including lower educated patients with no health coverage treated in public health centers and T2DM patients exclusively on oral antidiabetic drugs. We strongly feel that the assessment of patient-reported outcomes should be extended and incorporated into ambulatory practice and taken into account in the evaluation of diabetes care programs.

Even though Argentina has a National Diabetes Program (34) which supplies free insulin for all diabetes patients, clinical inertia and patient resistance are still barriers that delay insulin treatment. We expect that the results of this study will increase healthcare providers' awareness of patients' perceived QoL and help to overcome these barriers.

\section{Acknowledgments}

This work was financially supported by Sanofi-Aventis.

\section{Ethical issues}

The study protocol was approved by the institutional review board of the University Hospital and Center of Medical Education and Clinical Research "Norberto Quirno" (CEMIC for its acronym ins Spanish). Informed written consent was obtained from all participants in the study.

Competing interests

Authors declare that they have no competing interests.

\section{Authors' contributions}

$A P R, A A$, and $\mathrm{VI}$ contributed to the conception and design, with collaboration of $A B$. APR, VI, and AA coordinated the data collection. AA, with the collaboration of APR, CC, VI, and AB, conducted the analysis. CC and APR wrote the paper, with inputs from all authors. All authors contributed to subsequent and final drafts of the paper and approved its final version.

\section{Authors' affiliations}

${ }^{1}$ Institute for Clinical Effectiveness and Health Policy, Buenos Aires, Argentina. ${ }^{2}$ School of Public Health, University of Buenos Aires, Buenos Aires, Argentina. ${ }^{3}$ Servicio de Clínica Médica, Hospital Italiano de Buenos Aires, Buenos Aires, Argentina. ${ }^{4}$ Family and Community Medicine Division Hospital Italiano de Buenos Aires, Buenos Aires, Argentina.

\section{References}

1. Snoek FJ, Skinner TC. Psychology in diabetes care. 2nd ed. Chichester; Hoboken, NJ: Wiley; 2005.

2. Bradley C, de Pablos-Velasco P, Parhofer KG, Eschwege E, Gonder-Frederick L, Simon D. PANORAMA: a European study to evaluate quality of life and treatment satisfaction in patients with type-2 diabetes mellitus--study design. Prim Care Diabetes 2011; 5: 231-9. doi: 10.1016/j.pcd.2011.04.004

3. Koopmanschap M. Coping with Type II diabetes: the patient's perspective. Diabetologia 2002; 45: S18-22. doi: 10.1007/ s00125-002-0861-2

4. Rubin RR, Peyrot M. Quality of life and diabetes. Diabetes Metab Res Rev 1999; 15: 205-18. doi: 10.1002/(SICI)15207560(199905/06)15:3<205::AID-DMRR29>3.0.CO;2-O

5. Mata Cases M, Roset Gamisans M, Badia Llach X, Antoñanzas Villar F, Ragel Alcázar J. Impacto de la diabetes Mellitus tipo 2 en la calidad de vida de los pacientes tratados en las consultas de atención primaria en España (Effect of type-2 diabetes mellitus on the quality of life of patients treated at primary care consultations in spain). Aten Primaria 2003; 31: 493-9.

6. Jacobson AM. The psychological care of patients with insulindependent diabetes mellitus. N Engl J Med 1996; 334: 1249-53. doi: 10.1056/NEJM199605093341907

7. Hornquist JO, Wikby A, Stenstrom U, Andersson PO, Akerlind I. Type II diabetes and quality of life: a review of the literature. Pharmacoeconomics 1995; 8 Suppl 1: 12-6.

8. Aguiar CC, Vieira AP, Carvalho AF, Montenegro-Junior RM. [Assessment instruments for a Health-Related Quality of Life in diabetes mellitus]. Arq Bras Endocrinol Metabol 2008; 52: 931-9. [in Portuguese]. 
9. Norris SL. Health-related quality of life among adults with diabetes. Curr Diab Rep 2005; 5: 124-30.

10. Bradley C, Speight J. Patient perceptions of diabetes and diabetes therapy: assessing quality of life. Diabetes Metab Res Rev 2002; 18 Suppl 3: S64-9. doi: 10.1002/dmrr.279

11. Redekop WK, Koopmanschap MA, Stolk RP, Rutten GE, Wolffenbuttel BH, Niessen LW. Health-related quality of life and treatment satisfaction in Dutch patients with type 2 diabetes. Diabetes Care 2002; 25: 458-63.

12. Unden AL, Elofsson S, Andreasson A, Hillered E, Eriksson I, Brismar K. Gender differences in self-rated health, quality of life, quality of care, and metabolic control in patients with diabetes. Gend Med 2008; 5: 162-80. doi: 10.1016/j.genm.2008.05.003

13. Ferrante D, Linetzky B, Konfino J, King A, Virgolini M, Laspiur S. 2009 national Risk Factors survey: evolution of the epidemic of chronic non communicable diseases in Argentina. Cross sectional study. Rev Argent Salud Pública 2011; 2: 34-41.

14. Bradley C, Gilbride CJ. Improving treatment satisfaction and other patient-reported outcomes in people with type 2 diabetes: the role of once-daily insulin glargine. Diabetes Obes Metab 2008; 10 Suppl 2: 50-65. doi: 10.1111/j.1463-1326.2008.00871.x

15. Perrotta C, Irazola V. Validación linguistica de un cuestionario específico para medir calidad de vida relacionada con la salud (CVRS) "Audit of life diabetes dependent questionnaire" (ADDQOL) al español como se habla en la Argentina. Rev Soc Arg de Diabetes 2002; 36: 107.

16. Bradley C, Todd C, Gorton T, Symonds E, Martin A, Plowright $R$. The development of an individualized questionnaire measure of perceived impact of diabetes on quality of life: the ADDQoL. Qual Life Res 1999; 8: 79-91.

17. Gagliardino JJ, de la Hera M, Siri F. [Evaluation of the quality of care for diabetic patients in Latin America]. Rev Panam Salud Publica 2001; 10: 309-17. [in Spanish]

18. Ashwell SG, Bradley C, Stephens JW, Witthaus E, Home PD. Treatment satisfaction and quality of life with insulin glargine plus insulin lispro compared with NPH insulin plus unmodified human insulin in individuals with type 1 diabetes. Diabetes Care 2008; 31: 1112-7. doi: 10.2337/dc07-1183

19. DAFNE Study Group. Training in flexible, intensive insulin management to enable dietary freedom in people with type 1 diabetes: dose adjustment for normal eating (DAFNE) randomised controlled trial. BMJ 2002; 325: 746.

20. Polonsky WH. Emotional and quality-of-life aspects of diabetes management. Curr Diab Rep 2002; 2: 153-9.

21. Depablos-Velasco P, Salguero-Chaves E, Mata-Poyo J, DerivasOtero B, Garcia-Sanchez R, Viguera-Ester P. Quality of life and satisfaction with treatment in subjects with type 2 diabetes: results in Spain of the PANORAMA study. Endocrinol Nutr 2014; 61: 18-26. doi: 10.1016/j.endonu.2013.05.005

22. Hajos TR, Pouwer F, de Grooth R, Holleman F, Twisk JW, Diamant $\mathrm{M}$, et al. The longitudinal association between glycaemic control and health-related quality of life following insulin therapy optimisation in type 2 diabetes patients. A prospective observational study in secondary care. Qual Life Res 2012; 21: 1359-65. doi: 10.1007/s11136-011-0051-0

23. Weinberger M, Kirkman MS, Samsa GP, Cowper PA, Shortliffe EA, Simel DL, et al. The relationship between glycemic control and health-related quality of life in patients with non-insulindependent diabetes mellitus. Med Care 1994; 32: 1173-81.

24. Kuznetsov L, Long GH, Griffin SJ, Simmons RK. Are changes in glycaemic control associated with diabetes-specific quality of life and health status in screen-detected type 2 diabetes patients? Four-year follow up of the ADDITION-Cambridge cohort. Diabetes Metab Res Rev 2015; 31: 69-75. doi: 10.1002/ dmrr.2559

25. de Grauw WJ, van de Lisdonk EH, van Gerwen WH, van den Hoogen HJ, van Weel C. Insulin therapy in poorly controlled type 2 diabetic patients: does it affect quality of life? $\mathrm{Br} J$ Gen Pract 2001; 51: 527-32.

26. Glasgow RE, Ruggiero L, Eakin EG, Dryfoos J, Chobanian L. Quality of life and associated characteristics in a large national sample of adults with diabetes. Diabetes Care 1997; 20: 562-7.

27. Wang HF, Yeh MC. The quality of life of adults with type 2 diabetes in a hospital care clinic in Taiwan. Qual Life Res 2013; 22: 577-84. doi: 10.1007/s11136-012-0178-7

28. Alcubierre $\mathrm{N}$, Rubinat $\mathrm{E}$, Traveset $\mathrm{A}$, Martinez-Alonso $\mathrm{M}$, Hernandez M, Jurjo C, et al. A prospective cross-sectional study on quality of life and treatment satisfaction in type 2 diabetic patients with retinopathy without other major late diabetic complications. Health Qual Life Outcomes 2014; 12: 131. doi: 10.1186/s12955-014-0131-2

29. Spasić A, Radovanović RV, Đorđević AC, Stefanović N, Cvetković T. Quality of Life in Type 2 Diabetic Patients. Scientific Journal of the Faculty of Medicine in Niš 2014; 31: 193-200.

30. Collins MM, O'Sullivan T, Harkins V, Perry IJ. Quality of life and quality of care in patients with diabetes experiencing different models of care. Diabetes Care 2009; 32: 603-5. doi: 10.2337/ dc08-1169

31. Wee HL, Tan CE, Goh SY, Li SC. Usefulness of the Audit of Diabetes-Dependent Quality-of-Life (ADDQoL) questionnaire in patients with diabetes in a multi-ethnic Asian country. Pharmacoeconomics 2006; 24: 673-82.

32. Demirci H, Cinar Y, Bayram N, Bilgel N. Quality of life in type II diabetic patients in primary health care. Dan Med J 2012; 59: A4468.

33. Sundaram M, Kavookjian J, Patrick JH, Miller LA, Madhavan SS, Scott VG. Quality of life, health status and clinical outcomes in Type 2 diabetes patients. Qual Life Res 2007; 16: 165-77. doi: 10.1007/s11136-006-9105-0

34. Ministerio SyAS. Programa Nacional de Diabetes. PRONADIA, Resolución 301/1999; 1999. 